\title{
Growing hearts requires a beat
}

A team at Vanderbilt University (Nashville, TN) has made an important discovery regarding the early stages of heart valve formation. W. David Merryman directed the study, which examined the developing hearts of baby chickens to better understand how this key component of the heart is formed.

Eventually, the scientists hope to be able to grow replacement heart valves from a patient's own cells. Existing valves, made of plastic, do not grow with the patient and therefore need to be replaced multiple times in young patients, requiring repeated surgeries. "For the last 15 years, people have been trying to create a heart valve out of artificial tissue using brute-force engineering methods without any success," explained Merryman in a press release. "We decided to take a step back and study how heart valves develop naturally so we can figure out how to duplicate the process."

To do this, first author Mary Kathryn Sewell-Loftin devised a novel culture method for studying the mechanics of developing hearts removed from chick embryos. The U-shaped tube from which a heart develops

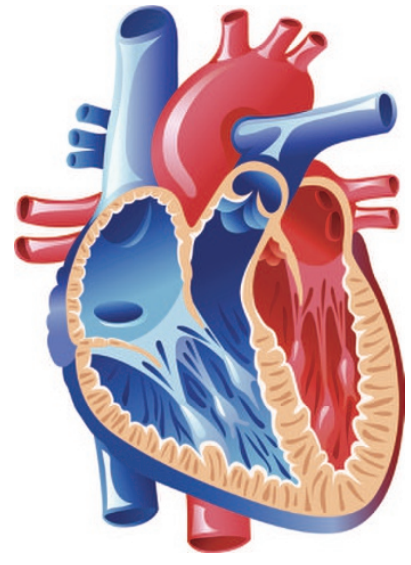

gel with a complex sugar called hyaluronic acid added.

Sewell-Loftin also created a computer program to measure the deformations the pulsing of the heart muscle cells created in the gel. The deformation maps showed that areas where there were stronger pulses, and as a result, stronger deformations, were the same areas where more valvular interstitial cells were being formed. This relationship demonstrates that the mechanical forces of the heart muscle on the cardiac jelly are essential for the process underlying the creation of heart valves (Biomaterials 35, 2809-2815; 2014).

"The discovery that the deformations consists of three layers: the outermost layer is made of cardiac muscle cells, the innermost layer is made of endothelial cells and the middle layer consists of a substance known as cardiac jelly. The cardiac jelly is essential to the process that turns the endothelial cells into valvular interstitial cells, which in turn guide the process of valve formation in the heart. Sewell-Loftin's culture method involved placing the heart in a dish filled with a cardiac jelly facsimile, consisting of collagen produced by the beating cardiac muscle cells are important provides an entirely new perspective on the process," Merryman said.

Next, the researchers are collaborating with stem cell scientists to develop the endothelial cells that comprise the inner layer of the embryonic heart. This step may be the missing piece for creating artificial heart valves made of human cells.

\section{Kara Rosania}

\section{MAPPING THE INSECT BRAIN}

An international consortium of neurobiologists called the Insect Brain Name Working Group recently released its magnum opus: a complete atlas of and consensus nomenclature for the structures of the arthropod brain (Neuron 81, 755-765; 2014). The group hopes that this resource will increase efficiency, encourage collaboration and enable comparison of studies among neuroscientists, leading to more meaningful interpretations of results.

Although insects such as fruit flies (Drosophila melanogaster) are used extensively in neurological and behavioral studies, descriptions and definitions of arthropod brain regions have been vague and variable, hindering analyses of the connections within the brain as well as attempts to draw links between its structure and function. Establishment and use of consistent terminology for brain anatomy is essential to progress in this area. And because insect brains are often considered a simpler model of human brains, insights developed from studying them could lead to improved understanding of our own brains.

To minimize confusion and enable such advances, the Insect Brain Name Working Group developed a systematic nomenclature for the insect brain, using the fruit fly as its primary reference but accounting for other arthropod taxa as well. Kei Ito (University of Tokyo, Japan) led the effort.

In creating the naming system, the consortium identified more than 50 anatomically distinct centers in the brain, or neuropils, including some that were previously un-named. This reflects a level of complexity similar to that of other animals like rodents and fishes, despite the diminutive size of the insect brain, which is comparable to that of a pinhead.

In naming the neuropils and other structures, the group chose terminology that would resolve existing ambiguities and retained classic terminology when possible. Most names refer to the shape or position of the structures. The group deliberately avoided using names with functional associations in recognition of the fact that future studies are likely to uncover new functions for the brain regions.

Nicholas Strausfeld (University of Arizona, Tucson), a co-author of the report, described the model in a press release. "This effort provides a three-dimensional road map for describing structures for all insect brains, and enables comparisons with other arthropods. It has huge value in describing network relationships between computational centers in the brain... By recognizing discrete centers in the insect brain, we will better understand how elaborations of the brains of insects and vertebrates might relate to each other despite more than 600 million years of divergent evolution." 